\title{
Prevenção de Aderências Pélvicas: Estudo Experimental em Ratas com Diferentes Modalidades Terapêticas
}

Pelvic Adhesion Prevention: Experimental Study on Rats with Different Therapeutic Agents José Fernando Pachecoํㅡㄹ Rogério Dias ${ }^{1}$, Márcia Guimarães da Silva², Andréa da Rocha Tristão ${ }^{1}$, Laurival Antônio De Luca ${ }^{1}$

\section{RESUMO}

Objetivos: avaliar o grau de aderências pélvicas em função do tempo e da utilização de diferentes substâncias empregadas na sua profilaxia.

Material e Métodos: estudo prospectivo com 120 ratas Wistar, albinas, virgens, 3 a 4 meses de idade, pesando aproximadamente 250 gramas, divididas aleatoriamente em 10 grupos de 12 animais cada: controle, sem lesão; lesões e sem tratamento; lesões + solução fisiológica 0,9\%; lesões + Ringer-lactato; lesões + dextrano 70 a 32\%; lesões + Ringer-lactato/heparina; lesões + Ringer-lactato/dexametasona; lesões + Ringer-lactato/hidrocortisona/ dexametasona/ampicilina; lesões + Ringer-lactato/albumina e lesões + carboximetilcelulose 1\%. Após anestesiados os animais, realizaram-se dois tipos de lesões nos cornos uterinos (escarificação e eletrocauterização), seguidos de tratamento profilático intraperitoneal com as soluções citadas. $N o 7^{\circ}, 14^{\circ}$ e $28^{\circ}$ dia pós-operatório, momentos $M_{1}, M_{2}$ e $M_{3}$, respectivamente, avaliaram-se quatro ratas de cada grupo quanto à presença de aderências. Os métodos empregados na quantificação das aderências encontradas basearam-se na classificação de Cohen, com escores variando de 0 a 4+ de acordo com a quantidade, caracteristicas $e$ localização das aderências. Foram usadas provas paramétricas para análise da variância e Kruskal-Wallis.

Resultados: os melhores tratamentos para prevenção de aderência pélvica em ratas foram: Ringer-lactato/dexametasona (predominio do escore 1+), dextrano 70 a 32\% (predominio do escore 2+) e Ringer-lactato/hidrocortisona/dexametasona/ampicilina (predominio do escore 2+). O periodo pós-operatório, representado pelo momento $M_{3}$, e a técnica cirúrgica, predominantemente com escore 0 , influíram na adesiólise e manutenção de aderências pélvicas em ratas.

Conclusões: a prevenção de aderências pélvicas em ratas inicia-se no processo cirúrgico de baixo dano tecidual; o uso de substâncias profiláticas (soluções) tem eficácia variada, sendo que algumas mostraram-se mais eficazes que outras.

PALAVRAS-CHAVE: Cirurgia experimental. Aderências pélvicas. Complicações da cirurgia.

Laboratório de Cirurgia Experimental do Departamento de Ginecologia e Obstetrícia - Faculdade de Medicina de Botucatu - UNESP, Botucatu - SP

${ }^{1}$ Departamento de Ginecologia e Obstetrícia, Faculdade de Medicina de Botucatu, UNESP

${ }^{2}$ Departamento de Patologia, Faculdade de Medicina de Botucatu, UNESP

Correspondência:

José Fernando Pacheco

Rua Acre, 183 - Vila Alexandria

15806-155 - Catanduva - SP

Fone: (17) 522-9564 / (17) 9775-4530

e-mail: jf_pacheco@uol.com.br

\section{Introdução}

Definem-se aderências peritoneais como sendo pontes de tecido fibroso neoformado que ligam duas ou mais estruturas anatomicamente separadas ${ }^{1}$. Podemos dividi-las em pélvicas, quando envolvem estruturas pélvicas, e abdominais, quando abrangem as demais estruturas intra-abdominais. Geralmente são seqüelas de cirurgias, doença inflamatória pélvica, apendicite, endometrio- 
se e corpos estranhos. Comumente inofensivas e assintomáticas, podem, contudo, alterar a anatomia e/ou função dos órgãos envolvidos, manifestando-se por meio de obstrução intestinal, dor pélvica, infertilidade e esterilidade ${ }^{2}$.

O peritônio é membrana de origem mesodérmica que reveste as cavidades abdominal, pélvica e os órgãos nelas contidos, e divide-se em peritôneo parietal e visceral. Macroscopicamente, trata-se de fina membrana transparente e frágil, revelando-se à microscopia como camada de tecido conjuntivo frouxo, coberta por única camada de células, o mesotélio ${ }^{3}$.

A homeostase peritoneal é mantida pelos vasos submesoteliais ${ }^{4}$ e por pequenos poros distribuídos pela superfície peritoneal, que permitem a remoção física passiva de substâncias. Contudo, outros mecanismos estão envolvidos na homeostase da cavidade peritoneal, como por exemplo, a fagocitose e o seqüestro (ou envolvimento) de material estranho por coágulo de fibrina ou pelo omento ${ }^{4}$.

A inflamação peritoneal (peritonite) ocorre em resposta a uma variedade de estimulos infecciosos (bactérias, virus, fungos e parasitas) e não infecciosos (traumas, cirurgias, agentes químicos e neoplasias $)^{2,5}$. No peritônio inflamado há permeabilidade exacerbada, o que resulta em aumento do volume do fluido peritoneal e na concentração de proteínas com liberação de substâncias quimiotáxicas e migração de leucócitos para o local.

A regeneração peritoneal é rápida e caracteriza-se por três fases: na primeira, ocorre reação inflamatória com infiltrado leucocitário e edema (semelhante aos outros tecidos); na segunda fase, a lesão é recoberta por exsudato de fibrina contendo hemácias e leucócitos; completando o processo cicatricial. Seguem-se os fenômenos de desbridamento, reparação e maturação de forma simultânea e não centrípeta. As células mesoteliais que recobrirão a lesão peritoneal originamse de células mesenquimais indiferenciadas, de fibroblastos do tecido conjuntivo subjacente, das células descamadas de outras vísceras e do peritônio parietal ${ }^{6}$.

Segundo Peacock ${ }^{6}$, uma série de fatores influencia o processo de cicatrização, tais como: espécie, porte do animal, sexo, traumas peritoneais, peritonite séptica, cirurgia prévia, suturas, aderências prévias, idade, má-nutrição, estresse crônico, uso de corticosteróides, entre outros.

O peritônio normal tem atividade fibrinolítica própria, decorrente da transformação do plasminogênio tecidual em plasmina. A sintese de plasmina é fundamental, já que esta substância é responsável pela dissolução da fibrina depositada na lesão, evitando, assim, a formação de aderência fibrosa ${ }^{3}$. O ativador do plasminogênio tecidual, principal catalisador da fibrinólise, é o responsável pela conversão de plasminogênio tecidual em plasmina. A inflamação e a isquemia reduzem a atividade fibrinolítica do peritônio devido a aumento local dos inibidores do ativador do plasminogênio tecidual tipo 1 e tipo 2 (PAI-1 e PAI-2) ${ }^{7}$. Acredita-se atualmente que a ocorrência de atividade fibrinolítica reduzida na cavidade peritoneal, alterando o balanço entre a coagulação e a fibrinólise, seja o mecanismo-chave para a formação de aderências peritoneais ${ }^{8}$.

$\mathrm{O}$ tratamento das aderências pélvicas e/ou abdominais é bastante controverso. Entretanto, minimizar sua formação secundária à laparotomia, empregando-se cuidados e técnicas cirúrgicas minimamente invasivas, é consenso atualmente ${ }^{8,9}$

Dentre as modalidades profiláticas mais utilizadas experimentalmente estão as aplicações intraperitoneais de: dextrano 70 a $32 \%^{10,11}$, carboximetilcelulose ${ }^{10,12}$, corticóides ${ }^{11}$ e antihistaminicos ${ }^{13}$. O uso profilático (pré e pós-operatório) de antibióticos de amplo espectro é recomendado $^{8,9}$, e o estímulo precoce da motilidade intestinal contribui para a profilaxia de aderências. Antiinflamatórios não esteróides seletivos mostraram-se também, experimentalmente, eficazes $^{11,13}$. Com o uso de suplementação com vitamina $\mathrm{E}$, evidenciou-se melhor resposta antiinflamatória ${ }^{6}$. Muitas dessas substâncias foram também empregadas em humanos ${ }^{14}$.

Recentemente, alguns autores têm avaliado o uso de membranas naturais, membranas artificiais $^{15,16}$ e cola de fibrina ${ }^{17}$ para recobrir a lesão peritoneal e prevenir a formação de aderências.

Por serem as aderências peritoneais quase inevitáveis no pós-operatório de cirurgias pélvicoabdominais, ocorrendo em mais de $60 \%$ dos casos, e apesar de apenas 30\% delas serem sintomáticas ${ }^{18}$, a sua associação, na mulher, com quadros de dor pélvica e principalmente infertilidade e esterilidade conjugal estimula a busca e o surgimento de meios cada vez mais eficazes na tentativa de evitá-las ou ao menos minimizá-las.

No presente estudo objetivamos avaliar a eficácia de diferentes substâncias empregadas rotineiramente e/ou experimentalmente na profilaxia das aderências peritoneais, bem como observar o grau de formação de aderências em função do tempo pós-cirúrgico.

\section{Material e Métodos}

Utilizamos 120 ratas virgens, Wistar, em idade de reprodução, que foram alojadas em caixas com no máximo cinco animais, à temperatu- 
ra de $25^{\circ} \mathrm{C}$, recebendo água e ração normal para animais de laboratório $\left(\right.$ Batavo $\left.^{\circledR}\right)$ ad libitum.

Os animais foram distribuídos aleatoriamente em 10 grupos experimentais delineados pelo procedimento cirúrgico com ou sem tratamento proposto (Tabela 1). Após anestesia, e de acordo com o grupo, os animais foram submetidos à laparotomia, com exposição dos cornos uterinos para a realização das lesões propostas: escarificação com gaze (corno direito) e eletrocauterização (corno esquerdo). Antes de se finalizar a sutura da cavidade peritoneal, instituiu-se tratamento profilático com $20 \mathrm{~mL}$ da solução preconizada para cada grupo (Tabela 1). Nos animais do grupo controle $(\mathrm{G}-\mathrm{C})$ apenas manipularam-se os órgãos pélvicos com pinças delicadas.

Tabela 1 - Grupos experimentais constituídos após o procedimento cirúrgico de acordo com o esquema terapêutico proposto ( $n=12$ para todos os grupos).

\begin{tabular}{ll}
\hline Grupos & Procedimento cirúrgico/terapêutico \\
\hline G-C & Grupo controle, sem lesão \\
GL-ST & Grupo com lesões, sem tratamento \\
GL-SF & Grupo com lesões + solução fisiológica 0,9\% \\
GL-D70 & Grupo com lesões + dextrano 70 a 32\% \\
GL-RL & Grupo com lesões + Ringer-lactato \\
GL-RLHep & $\begin{array}{l}\text { Grupo com lesões + Ringer-lactato + } \\
\text { heparina 2000 Ul/L }\end{array}$ \\
GL-RDL & $\begin{array}{l}\text { Grupo com lesões + ringer-Lactato + } \\
\text { dexametasona } 5 \mathrm{mg} / \mathrm{L}\end{array}$ \\
GL-RLHDA & $\begin{array}{l}\text { Grupo com lesões + Ringer-lactato + } \\
\text { hidrocortisona } 100 \mathrm{mg} / \mathrm{L}+ \\
\text { dexametasona } 5 \mathrm{mg} / \mathrm{L}+\text { ampicilina } 100 \mathrm{mg} / \mathrm{L}\end{array}$ \\
GL-RLAlb & $\begin{array}{l}\text { Grupo com lesões + Ringer-lactato + } \\
\text { albumina humana } 8 \mathrm{mg} / \mathrm{L}\end{array}$ \\
GL-CMC & Grupo com lesões + carboximetilcelulose 1\% $7 \mathrm{~mL} / \mathrm{kg}$ \\
\hline
\end{tabular}

GL-grupo com lesões

Após o procedimento cirúrgico, os animais de cada grupo foram divididos aleatoriamente em 3 subgrupos com 4 ratas, sendo cada um desses subgrupos estudados nos momentos $M_{1}\left(7^{\circ}\right.$ dia póscirúrgico), $\mathrm{M}_{2}$ (14\% dia pós-cirúrgico) e $\mathrm{M}_{3}\left(28^{\circ}\right.$ dia pós-cirúrgico), por meio de nova laparotomia e analisados, por um único observador em experimento duplamente cego, quanto ao grau de aderências pélvicas presentes, segundo a classificação de Cohen et al. ${ }^{19}$ modificada (Tabela 2).

Utilizamos para o referido estudo os seguintes métodos estatísticos: provas não paramétricas de Kruskal-Wallis e análise de variâncias paramétricas ${ }^{20}$, ambas com coeficiente de significância $\mathrm{p}$ menor que $5 \%$.
Tabela 2 - Critérios de classificação das aderências pélvicas segundo Cohen etal. ${ }^{19}$

\begin{tabular}{|c|c|}
\hline Escore & Características das lesões \\
\hline 0 & Ausência de aderências \\
\hline $1+$ & $\begin{array}{l}\text { Presença de uma única aderência fina, delgada, } \\
\text { localizada no local da lesão ou próxima a ele, } \\
\text { com extensão inferior a } 1,5 \mathrm{~cm}\end{array}$ \\
\hline $2+$ & $\begin{array}{l}\text { Presença de aderências finas, delgadas, localizadas } \\
\text { no local da lesão com extensão superior a } 1,5 \mathrm{~cm} \text {, } \\
\text { bilateralmente ou não }\end{array}$ \\
\hline $3+$ & $\begin{array}{l}\text { Presença de aderências delgadas, localizadas entre } 0 \\
\text { local da lesão e a parede abdominal e/ou } \\
\text { órgãos adjacentes }\end{array}$ \\
\hline $4+$ & $\begin{array}{l}\text { Presença de aderências densas, localizadas entre o } \\
\text { local da lesão e a parede abdominal e/ou } \\
\text { órgãos adjacentes }\end{array}$ \\
\hline
\end{tabular}

\section{Resultados}

No início do experimento, do ponto de vista clínico, todos os animais estavam livres de doenças. A antibioticoterapia profilática foi eficiente na proteção contra infecções durante o pós-operatório, com adequada cicatrização da ferida operatória. A técnica utilizada na obtenção de aderências pélvicas no modelo proposto (escarificação e cauterização) mostrou-se eficaz.

As 216 lesões uterinas provocadas resultaram em animais com os seguintes escores: 0: 14 $(12,9 \%) ; 1+: 28$ (25,9\%); 2+: 34 (31,6\%); 3+: 18 $(16,7 \%)$ e 4+: $14(12,9 \%)$, conforme mostra a Figura 1 . Observou-se entre todos os grupos maior predominância dos escores $1+$ e $2+(62 \%)$.

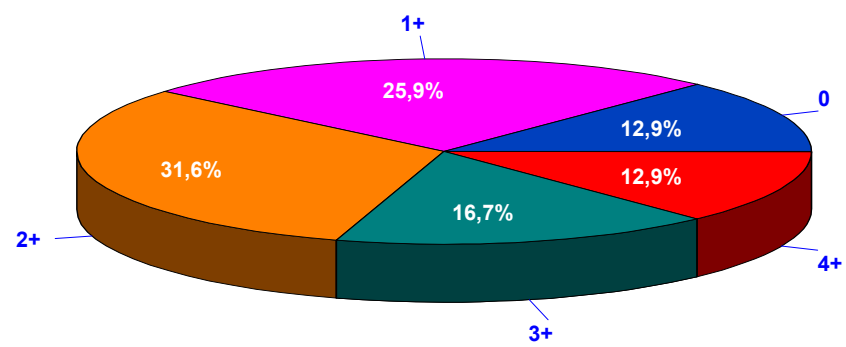

Figura 1-Freqüência total dos escores de aderências pélvicas nos grupos experimentais em todos os momentos do experimento.

O estudo estatístico pela análise de variâncias paramétricas da freqüência dos escores nos mostrou que os que apresentaram os melhores resultados, $\mathrm{p}<0,05$, foram: o grupo controle $\left(\mathrm{M}_{1}=\mathrm{M}_{2}=\mathrm{M}_{3}\right.$; escore $0>1+>2+=3+=4+$; $\mathrm{p}<0,05)$, o grupo lesão com Ringer-lactato + dexametasona $\left(\mathrm{M}_{1}=\mathrm{M}_{2}=\mathrm{M}_{3}\right.$; escore $1+>0=2+=$ $3+=4+; p<0,05)$, o grupo lesão com dextrano 70 
a $32 \%\left(M_{1}=M_{2}=M_{3}\right.$; escore $2+>0=1+=3+=4+$; $\mathrm{p}<0,05)$, o grupo lesão com Ringer-lactato + hidrocortisona + dexametasona + ampicilina $\left(\mathrm{M}_{1}\right.$ $=\mathrm{M}_{2}=\mathrm{M}_{3}$; escore 2+>0=3+=4+,1+>0=3+= $4+; \quad \mathrm{p}<0,05)$. Os resultados das freqüências dos animais desses grupos em relação aos escores observados em cada momento podem ser vistos na Tabela 3. Os demais grupos não apresentaram resultados diferentes.
A análise de cada grupo pelos testes não paramétricos de Kruskal-Wallis, de acordo com os momentos do experimento, mostrou-se significativa no momento $\mathrm{M}_{3}$ (escore $4+<0=1+=2+=3+$; $\mathrm{p}<0,05)$, no qual não se encontrou nenhum animal com escore 4+ (Tabela 3). O mesmo não pôde ser constatado na análise dos momentos $\mathrm{M}_{1}$ e $\mathrm{M}_{2}$.

Tabela 3 - Grupos experimentais após o procedimento cirúrgico e esquema terapêutico proposto relacionados com os escores ( 0 a $4+$ ) encontrados nos momentos $M_{1}$, $M_{2}$ e $M_{3}$ do experimento. Freqüência de animais (5) em relação aos escores observados.

\begin{tabular}{|c|c|c|c|c|c|c|c|c|c|c|c|c|c|c|c|}
\hline \multirow[b]{2}{*}{ Grupos } & \multicolumn{3}{|c|}{ Escore 0} & \multicolumn{3}{|c|}{ Escore 1+ } & \multicolumn{3}{|c|}{ Escore 2+ } & \multicolumn{3}{|c|}{ Escore 3+ } & \multicolumn{3}{|c|}{ Escore 4+ } \\
\hline & $M_{1}$ & $M_{2}$ & $\mathrm{M}_{3}$ & $M_{1}$ & $M_{2}$ & $\mathrm{M}_{3}$ & $M_{1}$ & $M_{2}$ & $\mathrm{M}_{3}$ & $M_{1}$ & $M_{2}$ & $M_{3}$ & $M_{1}$ & $M_{2}$ & $M_{3^{*}}$ \\
\hline G-S(*) & 75 & 75 & 75 & 25 & 25 & 25 & - & - & - & - & - & - & - & - & - \\
\hline GL-RLD") & - & - & - & 75 & 75 & 75 & - & 25 & 25 & 25 & - & - & - & - & - \\
\hline GL-D70") & - & - & 25 & 25 & 25 & - & 75 & 75 & 50 & - & - & 25 & - & - & - \\
\hline GL-RLHDA & - & - & 25 & 25 & 50 & 25 & 50 & 50 & 50 & - & - & - & 25 & - & - \\
\hline GL-ST & 25 & - & 50 & - & - & 25 & 25 & 25 & 25 & 25 & 25 & - & 25 & 50 & - \\
\hline GL-RLHep & 25 & 25 & - & 25 & 25 & - & 25 & 25 & 50 & - & - & 25 & 25 & 25 & - \\
\hline GL-RLAlb & 50 & - & 25 & 25 & - & 50 & - & 50 & - & - & 25 & 25 & 25 & 25 & - \\
\hline GL-SF & - & - & - & - & 25 & 25 & 25 & 50 & 25 & 50 & - & 50 & 25 & 25 & - \\
\hline GL-RL & 25 & - & 25 & - & - & - & - & 25 & 25 & 25 & 25 & 50 & 50 & 50 & - \\
\hline GL-CMC & - & 50 & - & 25 & 50 & 50 & - & - & 50 & 75 & - & - & - & - & - \\
\hline
\end{tabular}

(*) Resultados com significância estatística (p menor que 0,05$)$.

\section{Discussão}

A prevenção da formação de aderências pélvicas representa ainda grande desafio. As tentativas de evitá-las dependem de profundo conhecimento da sua fisiopatologia, do desenvolvimento e aprimoramento de técnicas cirúrgicas e da utilização de métodos preventivos. Estes compreendem desde a utilização de soluções com variadas substâncias ${ }^{19}$, até métodos de barreira ${ }^{15,16}$, sendo o uso de soluções o método mais estudado, difundido e utilizado.

No presente estudo, a técnica cirúrgica utilizada mostrou-se segura e eficaz, tanto na abordagem dos órgãos envolvidos ${ }^{19}$ quanto como modelo experimental para indução de aderências pélvicas (escarificação e cauterização). Não se observaram complicações de natureza infecciosa ou anestésica, embora possam ocorrer.

$\mathrm{O}$ escore mais freqüente foi o $2+$, ocorrendo em $31,6 \%$ dos animais, sendo este resultado diferente dos citados na literatura ${ }^{14}$, talvez porque nesses trabalhos, a metodologia empregada na produção das lesões tenha sido diferente.

Escores limitrofes, representados por 0 e 4+, totalizaram juntos $25,8 \%$ dos casos. Devemos lem- brar que em pequenos animais há fibrinólise muito ativa que limita a formação das aderências ${ }^{3}$.

Considerando os momentos do experimento em que se realizaram as análises das aderências $\left(7^{\circ}, 14^{\circ}\right.$ e $28^{\circ}$ dia pós-cirurgia), observamos que, no grupo G-C, 75\% dos animais não apresentaram aderências pélvicas e $25 \%$ tiveram aderências pélvicas com escore $1+$, independentemente do momento do experimento (não foram detectadas neste grupo aderências com escores $2+, 3+$ e 4+). Assim, na comparação da freqüência de escores dentro do grupo $\mathrm{G}-\mathrm{C}$, nota-se que o escore 0 foi predominante $(75 \%)$, seguido do escore $1+(25 \%)$.

Esses resultados sugerem que a técnica cirúrgica, quando realizada cuidadosamente, com menor grau de lesão, pode ser fator determinante na prevenção de aderências ${ }^{21}$.

No grupo GL-RLD, independente do momento da avaliação, não encontramos animais livres de aderências pélvicas. Contudo, nenhum animal teve escore $4+$. Neste grupo houve predomínio do escore $1+(75 \%)$, o mais freqüente também em todos os momentos do experimento. Cohen et al. ${ }^{19}$ observaram $55 \%$ de escores 0 a $2+$. Para esses escores, nossos resultados foram mais satisfatórios $(75 \%)$. A ação dos corticóides se deve em parte a 
sua habilidade em reduzir a permeabilidade vascular, estabilizando os lisossomos e reduzindo os efeitos subseqüentes das histaminas, impedindo a proliferação, migração e organização dos fibroblastos ${ }^{11,22}$.

No grupo GL-D70 observamos a ausência do escore 4+. Comparando-se a freqüência de escores nesse grupo, nota-se que o escore $2+$ foi o mais freqüente, independentemente do momento do experimento, sendo de $75 \%$ nos momentos $M_{1}$ e $M_{2}$ e $50 \%$ no momento $M_{3}$. A literatura apresenta trabalhos controversos, com resultados diferentes dos nossos. Graebe et al. ${ }^{10}$, em estudo realizado em ratas, encontraram $53 \%$ de animais sem aderência (escore 0 ), $7 \%$ com aderências leves (escores $1+$ e $2+$ ) e $33 \%$ com aderências graves (escores $3+$ e $4+$ ), o que se demonstrou de modo semelhante nos trabalhos de Elkins et $\mathrm{al}^{12}$. O dextrano 70 a $32 \%$ tem sido descrito como substância capaz de prevenir a formação de aderências por dois mecanismos: a hidroflotação, por meio da separação mecânica das superficies peritoneais lesadas, e a inibição química pela diminuição da deposição de fibrina (siliconização) ${ }^{11,23}$. É possível que casos de insucessos com o uso dessa substância se devam ao seu metabolismo, que envolve reações de hidrólise ácida e peritonite química.

Analisando o grupo GL-RLHDA constatamos que, independentemente do momento, quando comparada a freqüência de escores, $50 \%$ dos animais apresentaram escore $2+$, predominando sobre os demais. Considerando-se apenas o momento $\mathrm{M}_{2}, 50 \%$ deles tinham escore $1+$. Não encontramos escore $3+$. Estes resultados estão em concordância com a literarura ${ }^{14}$. Antibióticos associados a corticóides agiriam de modo bactericida, compensando o efeito imunossupressor do corticóide.

Considerando os momentos em que foram analisadas as aderências pélvicas, temos que, no momento $\mathrm{M}_{3}$ ( $28^{\circ}$ dia pós-cirurgia), houve predomínio de aderências pélvicas nos grupos G-C e GLST com o escore 0, nos grupos GL-RLD, GL-RLAlb e GL-CMC com o escore 1+, nos grupos GL-RLHep, GL-RLHDA, GL-D70 e GL-CMC para o escore 2+e nos grupos GL-SF e GL-RL com o escore 3+. Para o escore 4+ nenhuma aderência foi observada no momento $\mathrm{M}_{3}$. Neste caso, a análise estatística comparando a freqüência dos escores mostrou que o escore $4+$ foi significativamente menor que os demais.

A análise global dos momentos do experimento mostra que, no momento final do estudo $\left(\mathrm{M}_{3}\right), 28$ dias pós-cirurgia, há predomínio dos escores $0,1+$ e $2+$ e ausência do escore 4+. Esses dados mostram que, com o passar dos dias e independente do uso de determinadas substâncias na prevenção de aderências pélvicas, o tecido peritoneal exibe capacidade intrínseca responsável pela adesiólise. Outro fator que influencia na rapidez dessa adesiólise é a espécie e o tamanho dos animais (a velocidade com que ocorre a adesiólise é inversamente proporcional ao tamanho do animal) $)^{3,21}$.

Concluímos então que, em ratas, o uso de substâncias adjuvantes na prevenção de aderências pélvicas tem eficácia bastante variada; a prevenção de aderências pélvicas inicia-se no processo cirúrgico, no qual o dano tecidual é diretamente proporcional ao aparecimento de aderências pélvicas; os melhores tratamentos para prevenção de aderência pélvica em ratas, segundo o modelo proposto, foram: dextrano 70 a $32 \%$, Ringer-lactato + dexametasona e Ringer-lactato + hidrocortisona + dexametosona + ampicilina; os mecanismos endógenos de adesiólise peculiares a cada espécie influem no controle do aparecimento de aderências pélvicas no período pós-operatório.

\section{ABSTRACT}

Purpose: to evaluate the degree of pelvic adhesions in function of time and the different substances used in its prophylaxis.

Material and Methods: prospective study with 120 female, albino, virgin Wistar rats, 3 to 4 months of age, weighing approximately $250 \mathrm{~g}$, randomly divided into 10 groups of 12 animals each: control, without lesion; lesions and without treatment; lesions $+0.9 \%$ physiologic saline, lesions + Ringer lactate; lesions $+32 \%$ dextran 70 ; lesions + Ringer lactatel heparin; lesions + Ringer lactate/dexamethasone; lesions + Ringer lactate/hydrocortisone/dexamethasone/ampicillin; lesions + Ringer lactate/albumin, and lesions $+1 \%$ carboxymethylcellulose. The animals were anesthetized and two types of lesions (scarification and electrocauterization) were performed in the uterine horns, followed by treatment with the solutions, intraperitoneally, to prevent pelvic adhesions. On the 7th, 14th and 28th postoperative days, moments $M_{1}, M_{2}$ and $M_{3}$, respectively, the presence of adhesions was evaluated in 4 rats of each group. The methods applied to the quantification of the adhesions were based on Cohen's classification, with scores varying from 0 to $4+$ according to the amount, characteristics and location of the adhesions. Statistical analysis was performed by parametric tests for analysis of variance and the Kruskal-Wallis test. Results: the best treatments for prevention of pelvic adhesions in female rats were Ringer lactate/dexamethasone (score $1+$ prevalence), $32 \%$ dextran 70 to (score $2+$ prevalence) and Ringer lactate/hydrocortisone/dexamethasone/ampicillin (score $2+$ prevalence). The postoperative period, represented by moment $M_{3}$, and the surgical technique, predominantly with score 0, influenced adhesiolysis and maintenance of pelvic adhesions in female rats.

Conclusions: the prevention of pelvic adhesions in female rats begins with the surgical process at a smaller extent of 
tissue damage. The use of prophylactic substances (solutions) had a varied effectiveness, since some were more efficient than others.

KEYWORDS: Experimental surgery. Pelvic adhesions. Complications of surgery.

\section{Referências}

1. Holmdahl L, Risberg B, Beck DE, et al. Adhesions: pathogenesis and prevention-panel discussion and summary. Eur J Surg Suppl 1997; (577):56-62.

2. Ellis H. The clinical significance of adhesions: focus on intestinal obstruction. Eur J Surg Suppl 1997; (577):5-9.

3. Crowe DT, Bjorling DE. Peritoneum and peritoneal cavity. In: Slatter DH, editor. Textbook of Small Animal Surgery. $2^{\text {nd }}$ ed. Philadelphia: W. B. Saunders; 1993.p.407-30.

4. Trent AM. The peritoneum and peritoneal cavity. In: Kobluk CN, editor. The Horse Diseases Clinical Management. $1^{\text {st }}$ ed. Philadelphia: W. B. Saunders; 1995.p.373-404.

5. Santschi EM, Grindem CB, Tate LP Jr, Corbett WT. Peritoneal fluid analysis in ponies after abdominal surgery. Vet Surg 1988; 17:6-9.

6. Peacock EE Jr. Wound repair. $3^{\text {rd }}$ ed. Philadelphia: W. B. Saunders; 1984.p.526.

7. Vipond MN, Whawell SA, Thompson JN, Dudley HA. Peritoneal fibrinolytic activity and intra-abdominal adhesions. Lancet 1990; 335:1120-2.

8. Baxter GM. Intraabdominal adhesions in horses. Compend Contin Educ Pract Vet 1991; 13:1587-97.

9. Sullins KE. Intestinal adhesions reduction. In: White NA, editors. The equine acute abdomen. $1^{\text {st }}$ ed. Philadelphia: Lea \& Febiger; 1990.p.245-50.

10.Graebe RA, Oelsner G, Cornelison TL, Pan SB, Haseltine FP, De Cherney AH. An animal study of different treatments to prevent postoperative pelvic adhesions. Microsurgery 1989; 10:53-5.

11.Watson A, Vandekerckhove P, Lilford R. Liquid and fluid agents for preventing adhesions after surgery for subfertility. Cochrane Database Syst Rev 2000; (3):CD001298.
12.Elkins TE, Ling FW, Ahokas RA, Abdella TN, Homsey CA, Malinak LR. Adhesion prevention by solutions of sodium carboxymethylcellulose in the rat II. Fertil Steril 1984; 41:929-32.

13.Guvenal T, Cetin A, Ozdemir H, Yanar O, Kaya T. Prevention of postoperative adhesion formation in rat uterine horn model by nimesulide: a selective COX-2 inhibitor. Hum Reprod 2001; 16:1732-5.

14.Scott-Coombers DM, Vipond MN, Thompson JN. General surgeons' attitudes to the treatment and prevention of abdominal adhesions. Ann R Coll Surg Engl 1993; 75:123-8.

15.Farquhar C, Vandekerckhove P, Watson A, Vail A, Wiseman D. Barrier agents for preventing adhesions after surgery for subfertility. Cochrane Database Syst Rev 2000; (2):CD000475.

16.Dunn R, Lyman MD, Edelman PG, Campbell PK. Evaluation of the SprayGel adhesion barrier in the rat cecum abrasion and rabbit uterine horn adhesion models. Fertil Steril 2001; 75:411-6.

17.Meijer DW. Hemostats, adhesives, and sealants in endoscopic surgery. J Laparoendosc Adv Surg Tech A $2002 ; 12: 393-4$.

18.Peters AA, Trimbos-Kemper GC, Admiraal C, Trimbos JB, Hermans J. A randomized clinical trial on the benefit of adhesiolysis in patients with intraperitoneal adhesions and chronic pelvic pain. Br J Obstet Gynaecol 1992; 99:59-62.

19. Cohen BM, Heyman T, Mast D. Use of intraperitoneal solutions for preventing pelvic adhesions in the rat. J Reprod Med 1983; 28:649-53.

20.Curi PR. Metodologia e análise da pesquisa em ciências biológicas. $1^{\text {a }}$ ed. Botucatu: Tipomic; 1997.p.263.

21.Moreno A, Aguayo JL, Zambudio G, Ramirez P, Canteras M, Parrilla P. Influence of abdominal incision on the formation of postoperative peritoneal adhesions: an experimental study in rats. Eur J Surg 1996;162:181-5.

22.Treutner KH, Bertram P, Lerch MM, et al. Prevention of postoperative adhesions by single intraperitoneal medication. J Surg Res 1995; 59:764-71.

23.Diamond MP, De Cherney AH, Linsky CB, Cunningham T, Constantine B. Assessment of carboxymethylcellulose and 32\% dextran 70 for prevention of adhesions in a rabbit uterine horn model. Int J Fertil 1988; 33:278-82. 